\title{
Erratum to: Tauroursodeoxycholic Acid Protects Against Mitochondrial Dysfunction and Cell Death via Mitophagy in Human Neuroblastoma Cells
}

\author{
Inês Fonseca $^{1}$ - Gisela Gordino $^{1}$ • Sara Moreira ${ }^{1}$ • Maria João Nunes ${ }^{1} \cdot$ Carla Azevedo $^{1}$. \\ Maria João Gama $^{1,2}$ • Elsa Rodrigues ${ }^{1,2}$. Cecília Maria Pereira Rodrigues ${ }^{1,2}$. \\ Margarida Castro-Caldas ${ }^{1,3}$
}

Published online: 22 October 2016

(C) Springer Science+Business Media New York 2016

\section{Erratum to: Mol Neurobiol \\ DOI 10.1007/s12035-016-0145-3}

The original version of this article unfortunately contained a mistake at the Article Title. The first word "Tauroursodeoxycholic" was missing in the published paper.

The Article Title is hereby corrected to read "Tauroursodeoxycholic Acid Protects Against Mitochondrial Dysfunction and Cell Death via Mitophagy in Human Neuroblastoma Cells".

The original article was also corrected.

The online version of the original article can be found at http://dx.doi. org/10.1007/s12035-016-0145-3.

Margarida Castro-Caldas

mcastrocaldas@ff.ulisboa.pt

1 Research Institute for Medicines (iMed.ULisboa), Faculty of Pharmacy, Universidade de Lisboa, Av. Prof. Gama Pinto, 1649-003 Lisbon, Portugal

2 Department of Biochemistry and Human Biology, Faculty of Pharmacy, Universidade de Lisboa, Av. Prof. Gama Pinto, 1649-003 Lisbon, Portugal

3 Department of Life Sciences, Faculty of Science and Technology, Universidade NOVA de Lisboa, 2829-516 Caparica, Portugal 